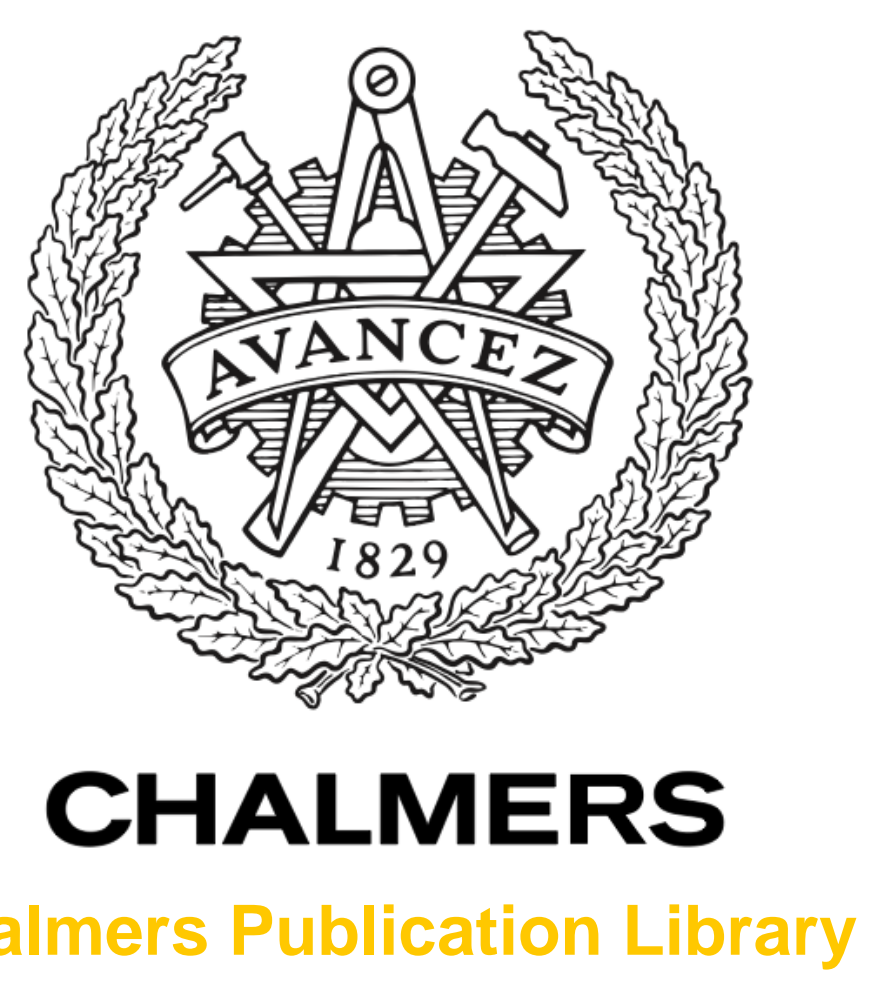

\title{
OTA characterization of BTS antenna in RIMP and random-LOS
}

This document has been downloaded from Chalmers Publication Library (CPL). It is the author's version of a work that was accepted for publication in:

\section{8th European Conference on Antennas and Propagation, EuCAP 2014, The Hague, The} Netherlands 6-11 April 2014

Citation for the published paper:

Gustafsson, M. ; Andersson, M. ; Chen, X. (2014) "OTA characterization of BTS antenna in RIMP and random-LOS". 8th European Conference on Antennas and Propagation, EuCAP 2014, The Hague, The Netherlands 6-11 April 2014 pp. 1394 - 1397.

http://dx.doi.org/10.1109/EuCAP.2014.6902040

Downloaded from: http://publications.lib.chalmers.se/publication/203784

Notice: Changes introduced as a result of publishing processes such as copy-editing and formatting may not be reflected in this document. For a definitive version of this work, please refer to the published source. Please note that access to the published version might require a subscription. 


\title{
OTA Characterization of $\mu$ BTS Antenna in RIMP and Random-LOS
}

\author{
Mattias Gustafsson ${ }^{1}$, Mats Andersson ${ }^{1}$, Chen Xiaoming ${ }^{2}$, Per-Simon Kildal ${ }^{2}$ \\ ${ }^{I}$ R\&D Center Huawei Technologies Sweden AB, Göteborg, Sweden \\ ${ }^{2}$ Signals and Systems Department, Chalmers University of Technology, Göteborg, Sweden \\ mattias.gustafsson@huawei.com,m.andersson@huawei.com, \\ xiaoming.chen@chalmers.se,per-simon.kildal@chalmers.se
}

\section{INTRODUCTION}

Huawei Technologies Sweden AB has been assigned the task of being a competence center to drive and invent new solutions and architectures in the area of base stations. A key to more effective base stations is new antenna technology. In the beginning of 2012, the Huawei Gothenburg office got the opportunity to start up a cooperation project with the Antenna Research group at Chalmers University of Technology.

One of the tasks of this project was to investigate a compact wideband antenna architecture to be used in a small cell environment. The other task of the project was to investigate how well this antenna performs with respect to some key parameters in the two reference environments RIMP and random-LOS. The RIMP (Rich Isotropic MultiPath) environment is well investigated and documented prior to the cooperation project [1]. The random-LOS environment is a more recent approach, that was introduced in [2] and studied for handheld wireless terminals in [3]. The work done in this cooperation is hence much of a pilot project to validate how well the key parameters can be investigated in such a models. The antenna used in this study is the wideband 4-port dual-polarized bowtie antenna described more extensively in [4], and the random-LOS characterization of it in [5]. During the study we found some fundamental limitations associated with how many ports small multiport antennas can have, and this is investigate in the parallel paper [6]. The numerical characterizations in these papers have been done by using the raybased simulation code described in [7].

\section{VALIDATION METHODS}

The RIMP channel is modelled by calculating the antenna terminal voltages when the antenna is exposed to a finite number of rays (k) arriving from random directions (Fig. 1). That is, each ray (k) has a random Angle of Arrival $\left.(\mathrm{AoA}) \varphi_{k} \sim U(0,2 \pi), \sin \theta_{k} \sim U(0, \pi)\right)$ that impinges into the antenna. Moreover, each incoming ray $(\mathrm{k})$ has an elliptical polarization with an axial ratio that may be any random number between 1 (i.e. circular) and $\infty$ (i.e. linear). Tilt angle and rotation direction is also random. Each ray also has a random propagation phase. If several antennas have exactly the same radiation pattern and exactly the same orientation, but different locations in the coordinate system, the AoA's and the polarizations of all incoming rays will be the same for the antennas, but there will still be a difference of the observed voltage on the terminals of the antennas because the propagation phase of each ray is different for each antenna due to their different positions.

The Random-LOS channel is simply modelled by limiting the number of rays (k) to just a single one (i.e. $\mathrm{k}=1$ ).

A channel realization is defined by the voltage measured on the antenna terminal when exposing the antenna to the $\mathrm{k}$ random rays generated by the RIMP and the random-LOS channels. If the incoming waves are normalized such that their mean power is equal to 1 , and we also select the threshold level of the receiver to be equal to 1 , the realizations of received powers will look something like Fig. 2.

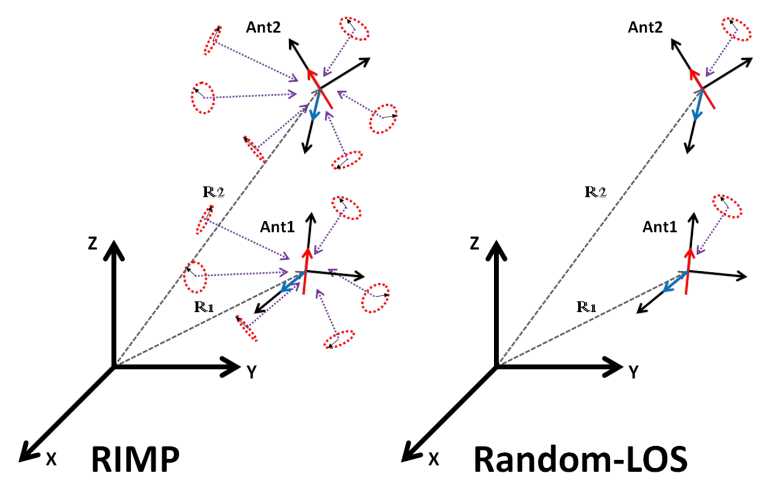

Figure 1: Illustration of how RIMP and Random-LOS cases are modelled.

With a threshold receiver, we say that we have detected the signal if the power of the received signal exceeds the threshold level. This means that all the realizations below $\mathrm{P}_{\mathrm{T}}$ are within the outage region and all the signals above $\mathrm{P}_{\mathrm{T}}$ are within the detection region (Fig. 2). If we sort the realizations $\mathrm{P}_{\mathrm{rn}}$ into a sorted set $\mathrm{P}_{\mathrm{rsn}}$ we can determine how much $\mathrm{P}_{\mathrm{AV}}$ would need to be increased to get a fraction of the realizations above $\mathrm{P}_{\mathrm{T}}$ (Fig. 3). The total number of realizations in the example illustrated is 19 , so this would mean that if we increase $\mathrm{P}_{\mathrm{AV}}$ by $\mathrm{P}_{\mathrm{T}} / \mathrm{P}_{\mathrm{rs} 3}$ (where $\mathrm{P}_{\mathrm{rs} 3}$ is the third sorted realization), then $3 / 19$ (approximately 16\%) of the realizations would remain below or on the detection threshold level.

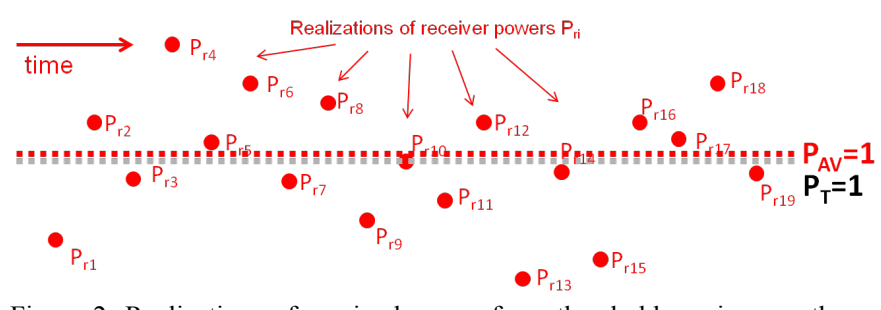

Figure 2: Realizations of received power for a threshold receiver, as they appear over time. 


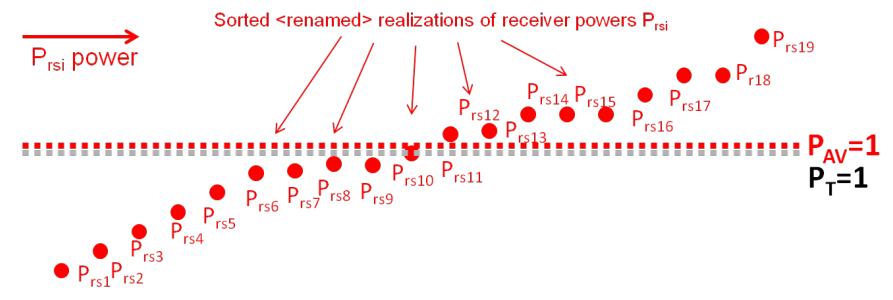

Figure 3: Realizations of received power for a threshold receiver, sorted.

If we make a CDF curve (Fig. 4) of $\mathrm{P}_{\mathrm{T}} / \mathrm{P}_{\mathrm{rsn}}$, we can read out how large the outage becomes if we would change $\mathrm{P}_{\mathrm{AV}}$ by a factor of $\mathrm{P}_{\mathrm{T}} / \mathrm{P}_{\mathrm{rsn}}$. In the figure we see the example of changing $\mathrm{P}_{\mathrm{AV}}$ by $\mathrm{P}_{\mathrm{T}} / \mathrm{P}_{\mathrm{rs} 17}$. This would essentially move all realizations of Fig. 3 downwards until $\mathrm{P}_{\mathrm{rs} 17}$ lands on the $\mathrm{P}_{\mathrm{T}}$-threshold, i.e. the only realizations that stays above PT are $\mathrm{P}_{\mathrm{rs} 17}, \mathrm{P}_{\mathrm{rs} 18}, \mathrm{P}_{\mathrm{rs} 19}$, and the outage probability hence becomes 16 realizations out of $19 \approx 84 \%$.

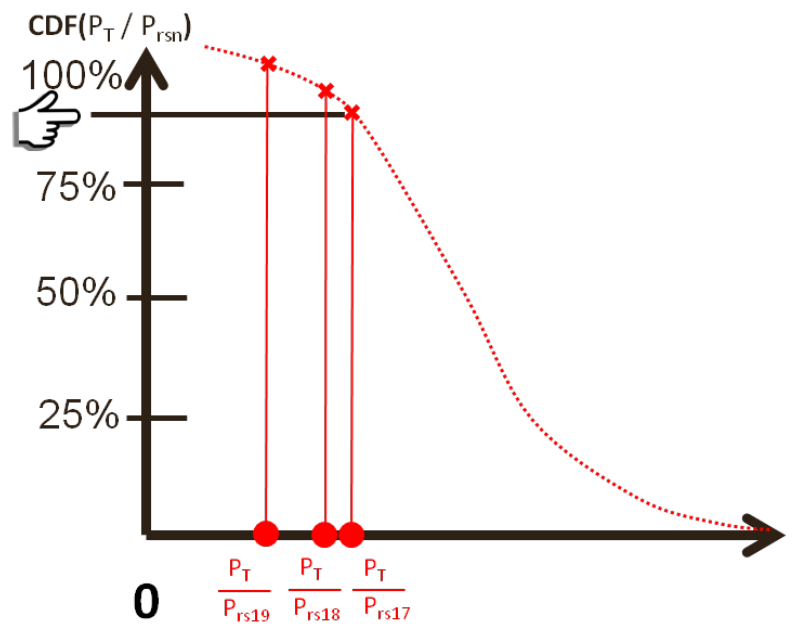

Figure 4: CDF curve of $\mathrm{P}_{\mathrm{T}} / \mathrm{P}_{\mathrm{rsn}}$ levels

If the outage probability is given by the $\operatorname{CDF}\left(\mathrm{P}_{\mathrm{T}} / \mathrm{P}_{\mathrm{rsn}}\right)$ then we easily realize that the detection probability is given by $1-\operatorname{CDF}\left(\mathrm{P}_{\mathrm{T}} / \mathrm{P}_{\mathrm{rsn}}\right)$, or more shortly $\operatorname{CCDF}\left(\mathrm{P}_{\mathrm{T}} / \mathrm{P}_{\mathrm{rsn}}\right)$. This finally leads us to the conclusion that the throughput for the system is given by

$\mathrm{T}=\mathrm{T}_{\max } \cdot \operatorname{CCDF}\left(\mathrm{P}_{\mathrm{T}} / \mathrm{P}_{\mathrm{rsn}}\right)$.

So far we have spoken about the detection probability for the case of one single antenna port. When dealing with MIMO systems, we have several antennas, which lead to that we get a unique set of realizations per antenna port. With the RIMP and the random-LOS channels we can generate the H-matrix (Eq. 2) which describes the relation of the observed signals on the receiver side compared to the transmitted signals on the transmitter side. The observed signals on the receiver side also include some noise that is introduced during the transmission.

$\mathbf{y}=\mathbf{H} \cdot \mathbf{x}+\mathbf{n}$

In the study performed in this work, the MIMO streams are separated by the methods of ZF (Zero Forcing on the receiver side) and SVD (Singular Value Decomposition). The former does not require any channel knowledge on the transmitter side, whereas the latter requires that the transmitter has channel knowledge.

Concerning the $\mathrm{ZF}$ detector, this is implemented by creating a detector based on the pseudo inverse of the H-matrix, acc. to Eq. (3).

$\mathbf{D}_{\mathrm{ZF}}=\left(\mathbf{H}^{\mathrm{H}} \cdot \mathbf{H}\right)^{-1} \cdot \mathbf{H}^{\mathrm{H}}$

If we multiply the received signal with this detector, we get

$\mathbf{y}^{\prime}=\mathbf{D}_{\mathrm{ZF}} \cdot \mathbf{y}=\left(\mathbf{H}^{\mathrm{H}} \cdot \mathbf{H}\right)^{-1} \cdot \mathbf{H}^{\mathrm{H}} \cdot(\mathbf{H} \cdot \mathbf{x}+\mathbf{n})=$

$\mathbf{x}+\left(\mathbf{H}^{\mathrm{H}} \cdot \mathbf{H}\right)^{-1} \cdot \mathbf{H}^{\mathrm{H}} \cdot \mathbf{n}$.

The received signal after using the $\mathrm{ZF}$ detector hence gives back $\mathbf{x}$, provided that the noise $\mathbf{n}$ is very low. Note that there is a risk of using the $\mathrm{ZF}$ detector for the case of low values in the H-matrix and large noise (i.e. low SNR). Then the noise will be amplified by the ZF detector, which will discriminate the received signal vector.

Provided that the transmitted vector $\mathbf{x}$ and the noise vector n consist of i.i.d. complex Gaussian elements with a variance of 1, the SNR of the i'th received stream is given by the reciprocal of the $i$ 'th element of the covariance matrix of the noise multiplied with the ZF-detector, i.e.

$\mathrm{SNR}_{\mathrm{ZF}}=$

$1 / \mathrm{E}\left[\left(\left(\mathbf{H}^{\mathrm{H}} \cdot \mathbf{H}\right)^{-1} \cdot \mathbf{H}^{\mathrm{H}} \cdot \mathbf{n}\right) \cdot\left(\left(\mathbf{H}^{\mathrm{H}} \cdot \mathbf{H}\right)^{-1} \cdot \mathbf{H}^{\mathrm{H}} \cdot \mathbf{n}\right)^{\mathrm{H}}\right]_{\mathrm{ii}}=$

$1 /\left[\left(\mathbf{H}^{\mathrm{H}} \cdot \mathbf{H}\right)^{-1} \cdot \mathbf{H}^{\mathrm{H}} \cdot \mathrm{E}\left[\mathbf{n} \cdot \mathbf{n}^{\mathrm{H}}\right] \cdot \mathbf{H} \cdot\left(\mathbf{H} \cdot \mathbf{H}^{\mathrm{H}}\right)^{-1}\right]_{\mathrm{ii}}=$

$1 /\left[\left(\mathbf{H}^{\mathrm{H}} \cdot \mathbf{H}\right)^{-1} \cdot \mathbf{H}^{\mathrm{H}} \cdot \mathbf{H} \cdot\left(\mathbf{H} \cdot \mathbf{H}^{\mathrm{H}}\right)^{-1}\right]_{\mathrm{ii}}=$

$1 /\left(\left[\left(\mathbf{H} \cdot \mathbf{H}^{\mathrm{H}}\right)^{-1}\right]_{\mathrm{ii}}\right)$

where the expectation $\mathrm{E}$ is taken over all the noise realizations. $1 /\left[\left(\mathbf{H} \cdot \mathbf{H}^{\mathrm{H}}\right)^{-1}\right]_{\mathbf{i i}}$ hence expresses the SNR's for the i'th received signal stream for the case of using the ZF detector.

Concerning transmission with precoding/decoding using SVD, we first perform the SVD operation on the H-matrix to extract the precoding matrix $\mathbf{V}$ and the decoding matrix $\mathbf{U}$. $\mathbf{U}$ and $\mathbf{V}$ are unitary matrices which is convenient, because it means that the symbols transmitted on the transmitter antennas can be transmitted with the same amplitude.

$[\mathbf{U}][\mathbf{S}]\left[\mathbf{V}^{\mathrm{H}}\right]=\mathbf{H}$

If we replace $\mathrm{H}$ by the components that we get out of the SVD operation, we get 
$\mathbf{y}=[\mathbf{U}][\mathbf{S}]\left[\mathbf{V}^{\mathrm{H}}\right] \cdot \mathbf{x}+\mathbf{n}$.

If we precode $\mathbf{x}$ with $\mathbf{V}$, and decode $\mathbf{y}$ with $\mathbf{U}^{\mathbf{H}}$, we get

$\mathbf{U}^{\mathrm{H}} \mathbf{y}=\mathbf{U}^{\mathrm{H}} \cdot \mathbf{U} \cdot \mathbf{S} \cdot \mathbf{V}^{\mathrm{H}} \cdot \mathbf{V} \cdot \mathbf{x}+\mathbf{U}^{\mathrm{H}} \cdot \mathbf{n}=$

$\mathbf{S} \cdot \mathbf{x}+\mathbf{U}^{\mathrm{H}} \cdot \mathbf{n}$.

Since $\mathbf{U}^{\mathrm{H}}$ is unitary, it will not amplify the noise, so the SVD is not dangerous in the same sense as the ZF detector when it comes to noise amplification.

Out of the methods above, we can determine the SNR needed to receive a number of parallel multiplexed streams.

\section{DEVELOPMENT OF THE BOWTIE ANTENNA}

By having the above models developed and verified, it was possible to evaluate how well different antennas perform in these reference environments. This was done both by means of theoretical modelling in the ViRMlab software Error! Reference source not found, and also by means of measurements of RIMP performance in reverberation chambers, and LOS performance in anechoic chambers. The Zero-Forcing (ZF) detector was applied on the received signal to verify the number of antenna streams that can be multiplexed with the antenna type analyzed. For the conducted project, the bowtie antenna (Fig. 2) outperformed other alternatives concerning the parameters of interest, like throughput and rank, when taking compactness and wide bandwidth $(1.7$ to $2.7 \mathrm{GHz})$ into account. The throughput corresponds to probability of detection (PoD) when normalized to unity, as shown in Fig. 5 for different number of bit streams for the i.i.d. cases.
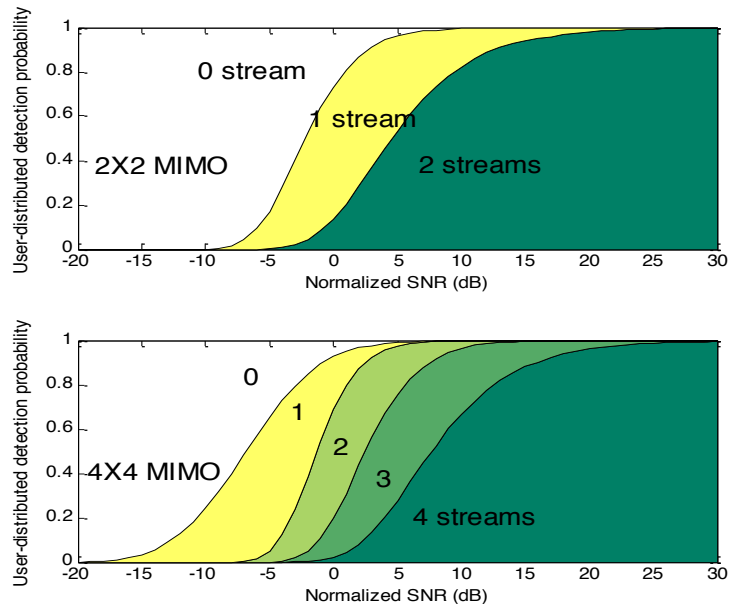

Figure 5: Multi-stream probability of detection (PoD) for i.i.d. case.

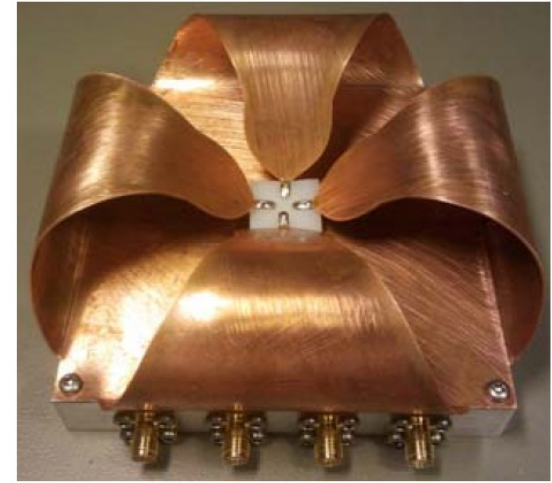

Figure 6: The 4-port bowtie antenna prototype.

The reason why the Bowtie antenna performs so well compared to other alternatives is due to the shape of its radiation (Fig. 8) patterns. Each port connects to a petal (Fig. 8) that radiates like a Huygens source in a direction that squints off in the petal direction relative to the groundplane of the antenna, i.e. the beam from petal 1 squints in the positive $y$ direction, the beam from petal 2 squints in the negative $x$ direction and so on. This makes the four ports of the bowtie antenna cover up a complete hemisphere around the antenna. The antenna will easily achieve rank 3 in a RIMP environment and rank 2 in a random-LOS environment. This wasproven both in theoretical analysis as well an in practical field trials that was conducted in a test network at the premises of one of Huawei's customers. If two bowtie antennas are mounted back-to-back, the combined antenna will actually cover the complete sphere and will be able to multiplex several streams over the sphere.

\section{CONCLUSIONS}

The project has resulted in successful methods to investigate spatial multiplexing in the reference environments RIMP and Random-LOS, by means of Zero Forcing and SVD algorithms. Moreover, an antenna solution - the 4-port bowtie antenna - has proven to perform very well in both the two reference environments and in field trials and is hence considered to perform very well in small cell BTS environments, when taking bandwidth and compactness into account. 


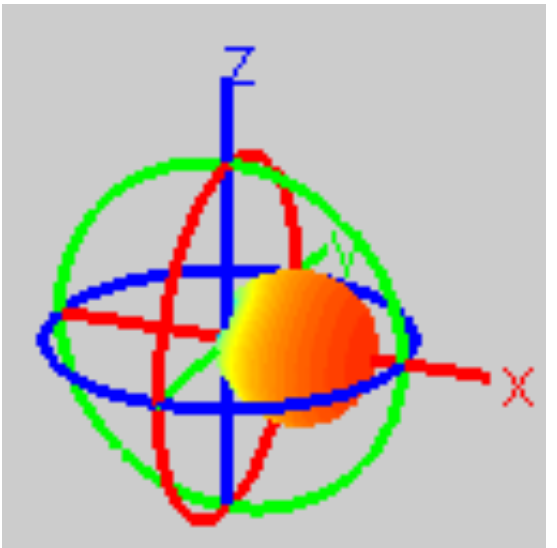

Figure 7: Huygens source radiation pattern.
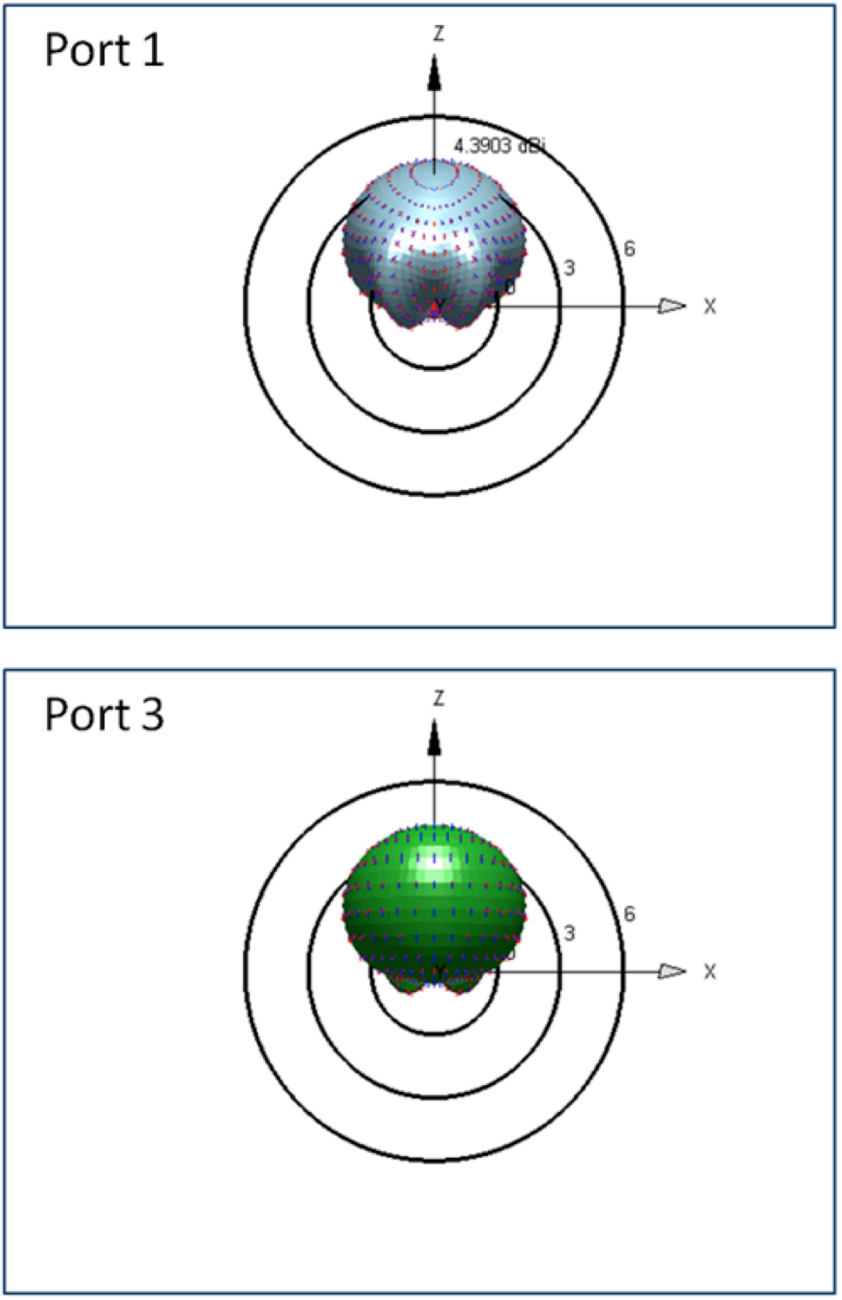

Figure 7: Single port radiation patterns of the Bowtie antenna.

\section{REFERENCES}

[1] X. Chen, P.-S. Kildal, J. Carlsson, and J. Yang, "MRC Diversity and MIMO Capacity Evaluations of Multi-Port Antennas Using Reverberation Chamber and Anechoic Chamber", IEEE Trans. Antennas Propag., vol. 61, No. 2, pp 917-926, February 2013.

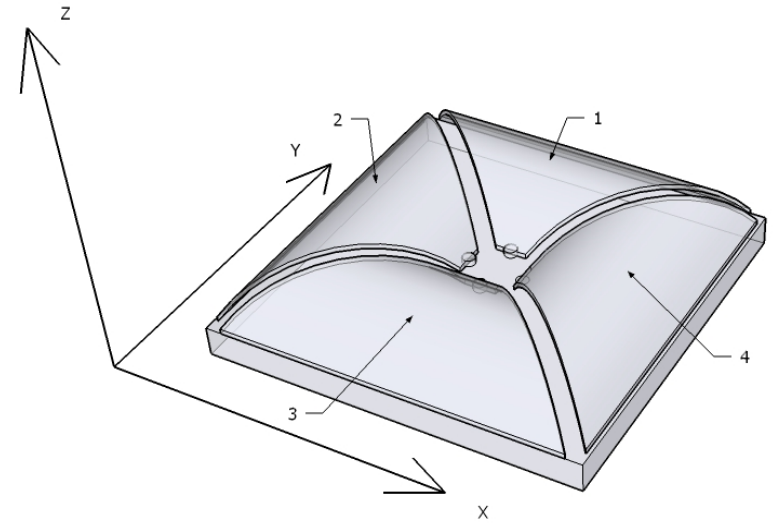

Figure 8: The four petals of the Bowtie antenna.
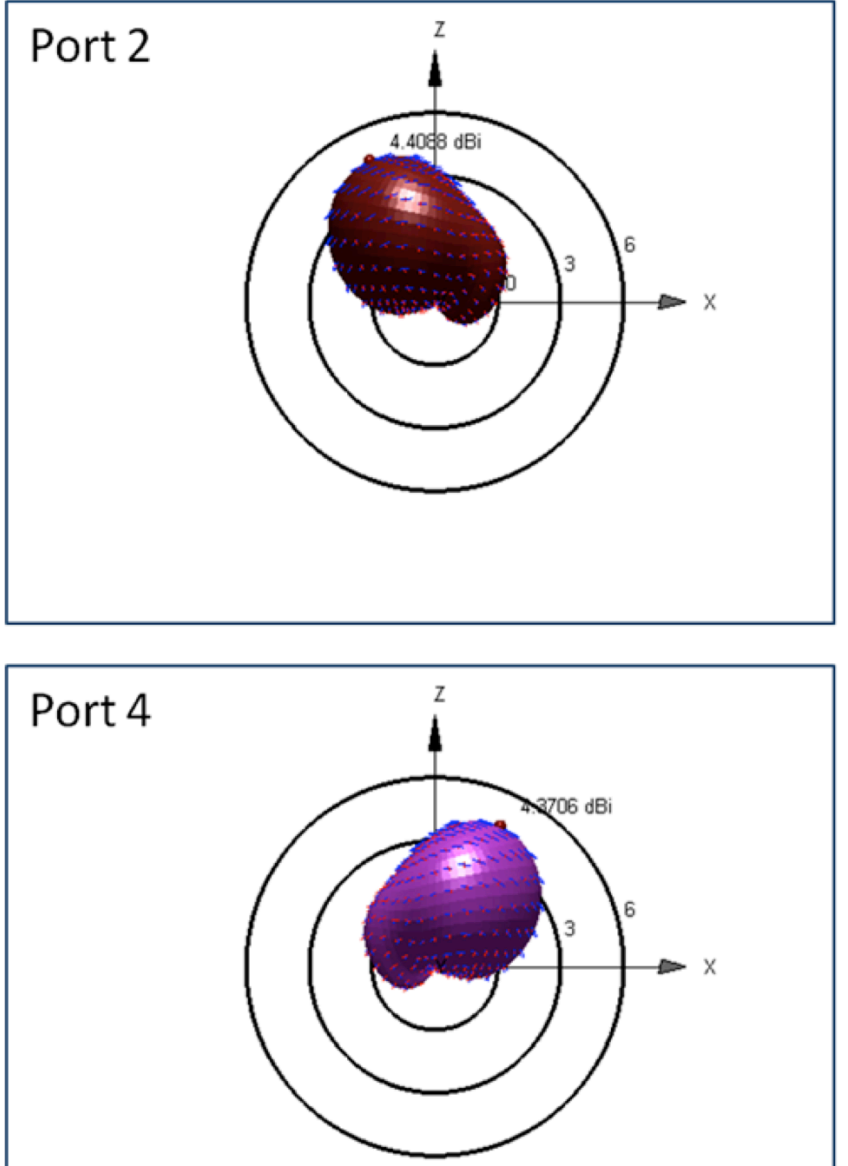

[2] P.-S. Kildal, C. Orlenius, and J. Carlsson, "OTA testing in multipath of antennas and wireless devices with MIMO and OFDM", Proc. IEEE, vol. 100, no. 7, pp. 2145-2157, July 2012.

[3] P.-S. Kildal, U. Carlberg, J. Carlsson, "Definition of Antenna Diversity Gain in User-Distributed 3D-Random Line-Of-Sight", Journal of Electromagnetic Engineering and Science (JEES), Vol. 13, No. 2, pp. 86-92, June 2013. 
[4] H. Raza, A. Hussain, J. Yang and P.-S. Kildal, "Wideband Compact 4-port Dual Polarized Self-grounded Bowtie Antenna", submitted to IEEE Transactions on Antennas and Propagation, 18th december, 2013.

[5] P.-S. Kildal, X. Chen, H. Raza, M. Gustafsson, Z. Shen, "Characterization of a Wideband Compact 4-port Bowtie Antenna for Micro BTS in Rich Isotropic Multipath and random-LOS", submitted to IEEE Transactions on Antennas and Propagation, Dec. 2013.
[6] P.S. Kildal, and X. Chen, "Fundamental Limitations on Small MultiBeam Antennas for MIMO Systems", in EUCap2014, Hague, Netherlands, 2014.

[7] U. Carlberg, J. Carlsson, A. Hussain, and P. S. Kildal, "Ray based multipath simulation tool for studying convergence and estimating ergodic capacity and diversity gain for antennas with given far-field functions" in 20th Internat. Conf. Applied Electromagnetics and Communications (ICECom), 20-23 Sept. 2010, Dubrovnik, Kroatia, 2010 